Collection SFN 12 (2011) 161-166

(C) Owned by the authors, published by EDP Sciences, 2011

DOI: $10.1051 / \mathrm{sfn} / 201112008$

\title{
Phonons calculated from first-principles
}

\author{
K. Parlinski
}

Institute of Nuclear Physics, Polish Academy of Sciences ul. Radzikowskiego 152, 31-342 Cracow, Poland

\begin{abstract}
First-principle phonon calculations can be performed using standard DFT program and the direct method. For that the supercell of studied crystal must be optimized, the Hellmann-Feynman forces derived, the dynamical matrix constructed and diagonalized, and hence all phonon modes calculated. The method has already been applied to large number of crystals, including crystals with defects and surfaces.
\end{abstract}

\section{INTRODUCTION}

Phonons determine the crystal temperature. A knowledge of phonon characteristics are required to describe mechanical, acoustic, dynamical, spectroscopic and thermodynamic properties of crystals at finite temperature. The easiest and perhaps most effective approach to calculate phonon frequencies and eigenvectors from first-principles is to use existing and well-tested softwares bases on the densityfunctional software (DFT) [1-4]. These computer codes can calculate crystal structures, electronic bands, magnetic properties, so called Hellmann-Feynman (HF) forces, and many other quantities. To avoid surface effects the DFT programs use the supercell concept. It means that only a fragment of a crystal is treated explicitly, and this fragment must be a multiplication of the primitive unit cell on which the three-dimensional periodic boundary conditions are imposed. The supercell contains then complete information on the crystal structure. The dynamical properties are extracted by computing the HF forces created by a displacement of one atom at a time or several atoms simultaneously. To find harmonic phonons it is sufficient to convert the response HF forces to a force constants and then to use the basic equations of lattice dynamics theory. This task is coded in the PHONON [5] computer software.

\section{DIRECT METHOD}

Usually, at $T=0$ the ground state energy $E$ as a function of atomic positions $\mathbf{R}(\mathbf{n}, \mu)$, where $\mathbf{n}$ is the primitive unit cell index and $\mu$ is the atomic index, is expanded over small displacements $\mathbf{U}(\mathbf{n}, \mu)$ up to harmonic term. Any atomic displacement $\mathbf{U}(\mathbf{m}, v)$ generates HF forces on all other atoms of the determined supercell according to the relationship

$$
F_{i}(\mathbf{n}, \mu)=-\sum_{\mathbf{m}, v, j} \Phi_{i, j}(\mathbf{n}, \mu, \mathbf{m}, v) U_{j}(\mathbf{m}, v)
$$

This relates the generated forces with the force constant matrices and atomic displacement. Knowing the displacements and arising HF forces one is able to determine the force constants $\Phi_{i, j}(\mathbf{n}, \mu, \mathbf{m}, \nu)$.

The dynamical matrix is defined as

$$
\mathbf{D}(\mathbf{k} ; \mu, v)=\frac{1}{\sqrt{M_{\mu} M_{v}}} \sum_{\mathbf{m}} \Phi(0, \mu ; \mathbf{m}, v) \exp \{-2 \pi i \mathbf{k} \cdot[\mathbf{R}(0, \mu)-\mathbf{R}(\mathbf{m}, v)]\}
$$

This is an Open Access article distributed under the terms of the Creative Commons Attribution-Noncommercial License 3.0, which permits unrestricted use, distribution, and reproduction in any noncommercial medium, provided the original work is properly cited. 
Here, summation $\mathbf{m}$ runs over all primitive unit cells of the crystal, $M_{\mu}, M_{\nu}$ are masses of atoms, and $\mathbf{k}$ is the wave vector. Diagonalization of the dynamical matrix

$$
\omega^{2}(\mathbf{k}, j) \mathbf{e}(\mathbf{k}, j)=\mathbf{D}(\mathbf{k}) \mathbf{e}(\mathbf{k}, j)
$$

gives the phonon frequencies $\omega^{2}(\mathbf{k}, j)$ and eigenvectors $\mathbf{e}(\mathbf{k}, j)$, called also polarization vectors.

The procedure of calculating the vibrational properties starts from minimizing the total energy of the crystal with respect to electronic degree of freedom, lattice constants and atomic positions At the minimized state all HF forces should vanish. Now, the HF forces should be calculated for configurations with single atoms displaced from the equilibrium position. The HF forces are calculated in the supercell with periodic boundary conditions. Now, consider a supercell in which one displace an atom $(\mathbf{m}, v)$ by $\mathbf{U}(\mathbf{m}, v)$. Due to periodic boundary conditions this displacement causes the same displacements of corresponding atoms $(\mathbf{m}+\mathbf{L}, v)$ in all images of the supercell. Here, $\mathbf{L}=\left(L_{a}, L_{b}, L_{c}\right)$ are the indices of lattice constants of the supercell. Thus, according to Eq. (2.1), a displacement of a single atom $(\mathbf{m}, v)$ in the original supercell generates a cummulant force which sums the force constants contributions from all supercell images. Thus, from the first-principle program one can only calculate the cummulant force constants. This requires that in the direct method one should try to use largest possible supercell, so that the contributions from atoms being in the image supercells are negligible. Practice shows that the supercell should have linear sizes of order of 5.0-7.0 A. [6, 7].

PHONON allows to determine the accuracy of phonon dispersion curves. For that one plots the values of the force constant parameters as a function of a distance between two atoms. Place the first atom in supercell center. Practice shows that if the values of the peripherals force constants, more precisely between central atom and periferial one, are less by three order of magnitude then the largest value of on-site force constant (action of the central atom on itself), i.e. $10^{-3}$, then the phonon dispersion curves are well reproduced for any wave vector. For smaller supercells one expects to obtain exact phonon frequencies for wave vectors which are commensurate with the supercell size and shape. In this case one has a few exact phonon frequencies and eigenvectors at exact wave vectors and interpolated phonon dispersion curves in the intermediate region. PHONON is also able to impose the translational-rotational invariance conditions causing the acoustic phonon modes to start at zero frequencies from the Brillouin zone center.

The program PHONOn solves Eq. (2.1) with respect to cummulant force constants which, in turn, are introduced to the dynamical matrix. Selected supercell should have a shape closest to a cube. Its symmetry should not break the crystal point group. All coordination shells defined within the supercell must have a complete list of atoms. Supercells of other shapes could reduce the crystal symmetry since the supercell acts as an external field. If it cannot be avoided to use supercell which breaks crystal symmetry, then a method described in [8] can recover the full crystal symmetry. Elongated supercell shapes can help to supplement the list of exact phonon frequencies calculated with the direct method along a particular crystal direction. For polar crystals the macroscopic electric field splits the infrared active phonon modes to LO and TO components. This splitting can be satisfactorily handled knowing the Born effective charges and the electronic dielectric constant. Moreover, the direct method allows to fit the computed phonon dispersion curves to desirable selected frequencies. This option can be used to build the most reliable phonon data based on first-principle and experimental methods.

\section{PHONON DISPERSION CURVES}

The VAsP [1, 2] and PHONON [5] programs have been applied to a number of crystalline systems. In the past the phonon dispersion curves and phonon density of states have been successfully calculated for $\mathrm{TiC}$ [9], $\mathrm{ZrC}$ [10], $\mathrm{MgO}$ [11], BN [12], GaN [8], HgSe [13], ZnTe [14], FeBO $\mathrm{O}_{3}$ [15], $\mathrm{AgGaS}$ [16], $\mathrm{AgGaSe}_{2}$ [17], AgGaTe $e_{2}, \mathrm{CuInS} \mathrm{I}_{2}$ [18], CuIn Se 2 [19], ZnSn P 2 [20], AgGaSe [21], CuInSe [22], CuInSe 2 [23], $\mathrm{Ba}_{8} \mathrm{Si}_{46}$ [24], $\mathrm{Ba}_{24} \mathrm{Si}_{100}$ [25], $\mathrm{UCoGa} a_{5}$ [26], PuCoGa 5 [27]. 
Some systems exhibit phase transitions and they often manifest themselves as soft modes. It occurs at $\mathrm{ZrO}_{2}$ [6], $\mathrm{GeO}_{2}$ [28], $\mathrm{SnO}_{2}$ [29], $\mathrm{LiNbO}_{3}$ [30], $\mathrm{MgSiO}_{3}$ [31], $\mathrm{CaTiO}_{3}$ [32]. Crystal with point defects can also be treated easily, like NiAl with Fe replacing Ni and Al [34]. Recent progress in handling the strong electron coupling by applying the $\mathrm{LDA}+\mathrm{U}$, or GGA+U approach allowed to calculated satisfactory phonons in such crystals as $\mathrm{CoO}$ [35], $\mathrm{Fe}_{3} \mathrm{O}_{4}$, having a Vervey phase transition [36], iron containing mineral $\mathrm{Fe}_{2} \mathrm{SiO}_{4}$ [37], and $5 f$ electron systems $\mathrm{PuCoGa} 5$ [27, 38]. The DFT calculations are not limited to bulk systems. With a slightly larger computational effort one may calculate phonons for surfaces, example being $\mathrm{Fe}(100), \mathrm{Fe}(110)$ [39], $\mathrm{MgO}$ [40] surfaces, and multilayers such as $\mathrm{Fe} e_{m} / \mathrm{Au}_{n}$ [41], Fe/FeSi [42], or Fe monolayer on $W$ (110) surface [43]. Placing an atom on the surface one may find the vibration spectrum of adsorbed atoms.

The phonon studies could be useful in searching for appropriate materials having superconducting properties like $\mathrm{Mo}_{3} \mathrm{Sb}_{7}$ [44], being novel thermoeletric materials [45], or showing magnetocaloric effect, as MnAs [46]. The last material shows an unusual property, namely a soft mode that varies its frequency as the magnetic moment of Mn atom changes.

\section{FILTERS}

In many cases, specially in more complex crystals with hundreds of phonon branches, it might be helpful to know which atom vibrates in a given phonon dispersion branch. This message can be extracted from different functions, called filters, of phonon eigenvectors. These filters determine the degree of participation of a selected phonon mode in the event defined by the functional form of the filter. Each filter defines some intensity of phonon mode. All proposed filters give non-negative values. Filter amplitude of a given mode is shown by colour. Analyzis performed with filters leads to deep understanding of the atomic displacement configurations involved in particular phonons. It allows to find correlations between other phenomena present in the crystal and phonons. Three filters described below, are proposed in PHONON.

\subsection{Atomic participations}

One may say how much a given atom $\mu$, displaced along $i=x, y$ or $z$, is involved in the $j$ phonon branch at wave vector $\mathbf{k}$ calculating the quantity:

$$
\sum_{\mu, i}\left|\mathbf{e}_{i}(\mathbf{k}, j ; \mu)\right|^{2}
$$

This quantity shows the square of vibrational amplitude of each atom and degree of freedom on the dispersion curve. The summation over different degrees of freedom is performed in the incoherent way. Thus, the phases of the eigenvectors are not taken into account. The intensities are independent of the selection of the Brillouin zone. Use this option to see the contribution of a given atom(s) to a chosen phonon branch. Summation over all atomic and coordinates of Eq. (4.1) converts this filter to orthonormality condition for the eigenvectors.

\subsection{Phonon intensities}

Phonon represents a coherent vibration of many atoms. One could group several atoms and degrees of freedom and study a coherent contribution of these atoms to the phonon branch. The corresponding filter looks like:

$$
\left|\sum_{\mu, i} \frac{\mathbf{e}_{i}(\mathbf{k}, j ; \mu)}{\sqrt{M_{\mu}}}\right|^{2}
$$


This filter follows from the next filter as a result of orientational averaging of the scalar product $\mathbf{k} \cdot \mathbf{e}_{i}(\mathbf{k}, j ; \mu)$. This quantity indicates the real occupation of the phonon branches. It takes into account the phases of the polarization vectors. It depends strongly on the Brillouin zone. Let us consider the simple example of a linear chain. Describe this chain in two ways, $A$ as a unit cell with single atom, and $B$ with twice lattice constant, and two atoms in the unit cell. The $A$ chain shows three phonon branches, while $B$ chain has six phonon branches, but the Brillouin zone is twice shorter. If you apply the filter, Eq. (4.2), we get identical results for $A$ and $B$ unit cells. It reflects the obvious fact that the phonon description should not depend on the selection of the unit cell. The option, Eq. (4.2), could be specially useful in the studies of point defects, surfaces, etc. when one must use large supercell consisting of several crystallographic unit cells, and when the formal symmetry is lower.

\subsection{Form factor}

The next filter is present in neutron coherent and $\mathrm{x}$-ray scatterings. It is defined as:

$$
\frac{1}{\mathbf{k}^{2}}\left|\sum_{\mu, i} \frac{\mathbf{k} \cdot \mathbf{e}_{i}(\mathbf{k}, j ; \mu)}{\sqrt{M_{\mu}}}\right|^{2}
$$

It shows the projection of polarization vectors onto phonon wave vector $\mathbf{k}$.

\section{THERMODYNAMICS}

The first-principle phonon calculations are limited to $T=0 \mathrm{~K}$. Thermodynamic properties of the crystals are to a large extent determined by phonons. The quasiharmonic approximation can describe such properties quite satisfactorily. This approach replaces in some sense the temperature changes of the crystal properties by the pressure dependence at $T=0 \mathrm{~K}$. In particular, a change of a crystal volume under increasing temperature is replaced by a similar change of a volume caused by a decrease of an ambient pressure. The approach to find the scaling factor between the pressure and temperature is the essence of the quasiharmonic approximation.

The Gibbs free energy $G(T, P)=F(T, V)+P V$ consists of the free energy $F(T, V)$ and $P V$ term. The free energy contains two contributions $F(T, V)=E(V)+F_{p h}(T, V)$, where $E(V)$ stands for the ground state energy and $F_{p h}(T, V)$ is the phonon free energy at a given unit cell volume $V$. The phonon free energy can be described by the following equation:

$$
F_{p h}(V, T)=k_{B} T \int_{0}^{\infty} g(\omega) \ln \left[2 \sinh \left(\frac{\hbar \omega}{2 k_{B} T}\right)\right] d \omega,
$$

where $\omega=\omega(V)$ denotes the volume dependent phonon frequencies and $g(\omega)$ is the phonon density of states.

To apply the quasiharmonic approximation one should proceed as follow [33]: (i) The free energy $F(T, V)$ should be calculated for several volumes $V$ of the unit cell. Temperature $T$ appears in $F(T, V)$ via the phonon term. (ii) One should construct the Gibbs free energy $G(T, P)$ by adding to the free energy the term $P V$, where $P$ is the desired pressure, and $V$ is the volume for which $F(T, V)$ was calculated. (iii) The locus of the minima of $G(T, P)$ for a fixed pressure $P$ determines the stable states at a given temperature $T$. For a fixed pressure $P$ these minima of $G(T, V)$ should be presented as plots of a temperature dependence of the Gibbs free energy. Notice that the main contribution to a decrease of the Gibbs free energy as a function of temperature originates from the phonon free energy term. (iv) A state with the lower Gibbs free energy at a given temperature $T$ and pressure $P$ represents the stable phase. A crossing point of two curves representing different structures denotes the first order phase transition with coexistence temperature $T_{c}$ and pressure $P_{c}$. 
Combining $\left(T_{c} P_{c}\right)$, the critical temperatures and pressures, the P-T phase diagrams were established in the quasiharmonic approximation. In this way the $\mathrm{P}-\mathrm{T}$ phase diagrams of $\mathrm{MgSiO}_{3}$ [47], and $\mathrm{AlN}$ [33] have been found. It is worth noting that a strong first-order phase transition can be more easily located on the P-T surface than phase transitions that are close to second order. The limited analysis of the free energy temperature dependence provides the temperature of the phase transitions in $\mathrm{ZrO}_{2}$ [48] and $N i T i$ [49].

\section{FINALE REMARKS}

The direct method has some advantages and disadvantages. A great advantage of the direct method is that it could use the data calculated by any standard DFT program, which is able to calculate the HF forces. For complex crystals for which the supercell is as big as the unit cell, and for which the interaction range is confined to the supercell, the phonon frequencies could be well reproduced. A disadvantage is that within direct method it is not easy to calculate the LO/TO splitting, and that the largest error on phonon frequencies is expected for the low-energy modes.

There are still quantities which are difficult to handle by this type of phonon calculation. Heat conductivity depends crucially on the phonon relaxation time [50], which is determined by the anharmonic part of the crystal potential energy. Atomic diffusion can be handle with care when finding the transition state during the hoping process [51].

\section{References}

[1] G. Kresse and J. Hafner, Phys. Rev., 47, 558 (1993); ibid 49, 14251 (1994).

[2] G. Kresse and J. Furhtmüller, Phys. Rev. B 54, 11169 (1996). Software VAsP, Vienna (2002), http://cms.mpi.univie.ac.at/vasp/vasp.html.

[3] P. Blaha, K. Schwarz, G.K.H. Madsen, D. Kvasnicka and J. Luitz, Software WIEN2k, http://www.wien2k.at/

[4] E. Artacho, J. Gale, A. García, J. Junquera, P. Ordejón, D. Sánchez-Portal and J.M. Soler, Software SiESTA 2001, http://www.uam.es/siesta/

[5] K. Parlinski, Software PHONON, Cracow (2010), http://wolf.ifj.edu.pl/phonon/, http://www.ComputingForMaterials.com/

[6] K. Parlinski, Z.Q. Li and Y. Kawazoe, Phys. Rev. Lett. 78, 4063 (1997).

[7] K. Parlinski, Conference Proceedings 479, "Neutrons and Numerical Methods - $N_{2} M$ edited by M.R. Johnson, G.J. Kearley and H.G. Büttner, Am. Inst. Phys. p. 121 (1999).

[8] K. Parlinski and Y. Kawazoe, Phys. Rev. B 60, 15511 (1999).

[9] P. T. Jochym, K. Parlinski and M. Sternik, Europ. Phys. J. B 10, 9 (1999).

[10] P.T. Jochym and K. Parlinski, Euro. Phys. J. B 15, 265 (2000).

[11] K. Parlinski, J. Łażewski and Y. Kawazoe, J. Phys. Chem. Solids, 61, 87 (1999).

[12] K. Parlinski, J. Alloys and Compounds 328, 97 (2001).

[13] J. Łażewski, K.Parlinski, W.Szuszkiewicz and B.Hennion, Phys. Rev. B 67, 134300 (2003).

[14] J. Camacho, K. Parlinski, A. Cantarero and K. Syassen, Phys. Rev. B 70, 033205 (2004).

[15] K. Parlinski, J. Łażewski, P.T. Jochym, A. Chumakov, R. Rüffer and G. Kresse, Europhysics Lett. 56, 2 (2001).

[16] J. Łażewski and K. Parlinski, J. Phys. Chem. 328, 162 (2001).

[17] J. Łażewski and K. Parlinski, J. Phys.: Condens. Matter 11, 9673 (1999).

[18] J. Łażewski, P.T. Jochym and K. Parlinski, J. Chem. Phys., 117, 2726 (2002).

[19] J. Łażewski, K. Parlinski, B. Hennion and R. Fouret, J. Phys.: Condens. Matter 11, 9665 (1999).

[20] J. Łażewski and K. Parlinski, J. Alloys and Compounds, 114, 6734 (2001). 
[21] R. Fouret, P. Derollez, A. Laamyem, B. Hennion and J. Gonzalez, J. Phys.: Condens. Matter, 9, 6579 (1997).

[22] P. Dorollez, R. Fouret, A. Laamyem, B. Hennion and J. Gonzalez, J. Phys.: Condens. Matter, 11, 3987 (1999).

[23] J. Łażewski, H. Neumann, K. Parlinski, G. Lippold and B.J. Stanbery, Phys. Rev., B 68, 144108 (2003).

[24] J.S. Tse, T. Iitaka, T. Kume, H. Shimizu, H. Fukuoka and S. Tamanak, Phys. Rev., 72, 155441 (2005)

[25] J.S. Tse, T. Iitaka and K. Parlinski, Phys. Rev., 75, 153 (2006).

[26] U. Metoki, K. Kaneko, S. Raymond, J.-P. Sanchez, P. Piekarz, K. Parlinski, A.M.Oleś, S. Ikeda, T.D. Matsuda, Y. Haga, Y. Onuki and G.H. Lander, Physics B, 378-380, 1003 (2006).

[27] P. Piekarz, K. Parlinski, P.T. Jochym, A.M. Oleś, J-P. Sanchez and J. Rebizant, Phys. Rev., 72014 $521(2005)$

[28] Z. Łodziana K. Parlinski and J. Hafner, Phys. Rev., B 63, 134106 (2001).

[29] K. Parlinski and Y. Kawazoe, Europ. Phys. J., B 13, 679 (2000).

[30] K. Parlinski, Z.Q. Li and Y.Kawazoe, Phys. Rev. B 61, 272 (2000).

[31] K. Parlinski and Y. Kawazoe, Europ. Phys. J., B 16, 49 (2000).

[32] K. Parlinski and Y. Kawazoe, J. Chem. Phys., 114, 2395 (2001).

[33] A. Siegel, K. Parlinski and U.D. Wdowik, Phys. Rev., B 74, 104116 (2006).

[34] K. Parlinski, P.T. Jochym, O. Leupold, A. Chumakov, R. Rüffer, H. Schober, A. Jianu, J. Dutkiewicz and W. Maziarz, Phys. Rev., 70224304 (2004).

[35] U.D. Wdowik and K. Parlinski, Phys. Rev., B bf 75104306 (2007).

[36] P. Piekarz, K. Parlinski and A.M. Oleś, Phys. Rev. Lett, 97, 156402 (2006).

[37] M. Derzsi, P. Piekarz, P.T. Jochym, J. Łażewski, M. Sternik, A.M. Oleś, and K. Parlinski, Phys. Rev., B 79, 205105. (2009).

[38] S. Raymond, P. Piekarz, J.-P. Sanchez, J. Serrano, M. Krisch, B. Janousova, J. Rebizant, N. Metoki, K. Kaneko, P.T. Jochym, A.M. Oleś and K. Parlinski, Phys. Rev. Lett., 96, 237003 (2006).

[39] J. Łażewski, J. Korecki and K. Parlinski, Phys. Rev. Lett., 75, 054303 (2007).

[40] K. Parlinski 2006 Phys. Rev. B 74, 184309 (2006).

[41] M. Sternik, K. Parlinski, J. Korecki, Phys. Rev., B 74, 195405 (2006).

[42] P.T. Jochym, K. Parlinski, and A.M. Oleś, Phys. Rev., B 73224411 (2006).

[43] J. Łażewski, P. Piekarz, A. M. Oleś, J. Korecki, and K. Parlinski, Phys. Rev., B 76205427 (2007).

[44] B. Wiendlocha, J. Tobola, M. Sternik, S. Kaprzyk, K. Parlinski, and A. M. Oleś, Phys. Rev., B 78, 060507 (2008).

[45] M.M. Koza, M.R. Johnson, R. Viennois, H. Mutka, L. Girard, and D. Ravot, Nature Materials 7, 805 (2008).

[46] J. Łażewski, P. Piekarz, J. Toboła, B. Wiendlocha, P.T. Jochym, M. Sternik, and K. Parlinski, Phys. Rev. Lett., 104147205 (2010).

[47] M. Sternik, K. Parlinski, J. Phys. Chem. Solids, 67, 796 (2006).

[48] M. Sternik and K. Parlinski, J. Chem. Phys., 122, 064707 (2005).

[49] K. Parlinski, M. Parlinska-Wojtan, Phys. Rev., B 66, 064307 (2002).

[50] Niko de Koker, Phys. Rev. Lett., 103, 125902 (2009).

[51] E. Wimmer, W. Wolf, J. Sticht, and P. Saxe, Phys. Rev. B 77, 134305 (2008). 